\title{
Cerebrospinal fluid approach on neuro-oncology
}

\author{
Estudo do líquido cefalorraquiano em neuro-oncologia
}

Hélio Rodrigues Gomes

\begin{abstract}
Central nervous system (CNS) involvement is a major complication of haematological and solid tumors with an incidence that ranges from $10 \%$ in solid malignances up to $25 \%$ in specific leukaemia or lymphoma subtypes. Cerebrospinal fluid (CSF) patterns are unspecific. Though CSF cytology has a high specificity (up to 95\%), its sensitivity is generally less than $50 \%$ and no diagnostic gold standard marker is available, yet. New technologies such as flow cytometry, molecular genetics and newer biomarkers may improve diagnostic sensitivity and specificity, leading to the CNS involvement diagnosis, and consequently, to an effective prophylaxis and successful treatment.
\end{abstract}

Keywords: cerebrospinal fluid, biomarkers, leptomeningeal metastasis, neoplastic meningitis.

\section{RESUMO}

O envolvimento do sistema nervoso central (SNC) é uma das maiores complicações das neoplasias de linhagem hematológica e dos tumores sólidos, com uma incidência que varia de 10\% nestes últimos até 25\% nas leucemias e subtipos de linfomas. Os padrões do líquido cefalorraquiano (LCR) nestes casos é inespecífico. Embora a citologia do LCR tenha uma alta especificidade (acima de 95\%), sua sensibilidade é geralmente menor que 50\%, e nenhum marcador biológico de padrão-ouro é disponível até o momento. Novas tecnologias, como a citologia de fluxo, a genética molecular e novos biomarcadores poderão aumentar a sensibilidade e especificidade no diagnóstico, levando ao diagnóstico de envolvimento do SNC, e consequentemente a profilaxia efetiva e tratamento bem sucedido.

Palavras-Chave: líquido cefalorraquiano, biomarcadores, metástase leptomeníngea, meningite neoplásica.

Diagnosis of central nervous system (CNS) involvement in neoplastic diseases is a major problem. Recent progresses in chemotherapies and targeted therapies have improved survival in cancer patients. In this context of better-controlled systemic disease, brain metastases (BM) are emerging as a new challenge for the oncologist ${ }^{1}$.

$\mathrm{BM}$ are one of the most common neurologic complications of cancer. The incidence is $9 \%-17 \%$ based on various studies, although the exact incidence is thought to be higher $^{2}$. The incidence is increasing with the availability of improved imaging techniques which aid early diagnosis, and effective systemic treatment regimens which prolong life, thus allowing cancer to disseminate to the brain. Lung cancer, breast cancer, and melanoma are the most frequent to develop brain metastases, and account for $67 \%-80 \%$ of all cancers. Some patients present with no known primary cancer diagnosis ${ }^{2,3}$.

Cancer cells may invade the meninges through different pathways, depending on histology of the primary tumor. The most common route of metastasis resulting in leptomeningeal metastasis (LM) is probably the hematogenous spread to the arachnoid via the arterial circulation, mainly in hematological malignancies ${ }^{4,5}$. Vertebral and paravertebral metastases (particularly from breast and lung cancers), as well as head and neck cancers, may spread centripetally along peripheral or cranial nerves via the endoneural/ perineural route or along coassociated lymphatics or veins gaining access through the dural and arachnoidal sleeves of nerve roots (spinal roots, cranial nerves) and subsequently into the subarachnoid space ${ }^{5,6}$.

Direct spread from brain parenchyma that is in close to the cerebrospinal fluid (CSF) space has been described. These tumors may breach the subarachnoid or ventricular spaces and diffuse widely in the CSF. This manner of spread is particularly relevant with respect to primary brain tumors. Primary tumors arising in the meninges such as melanoma and some soft tissue sarcomas (e.g., malignant peripheral nerve sheath tumors) may secondarily spread to the CSF and disseminate ${ }^{2}$. Another way of CSF tumor spread is that resulting during invasive procedures, through ependymal or pial breach ${ }^{2,6}$. 
Once malignant cells enter the CSF, they disseminate along the meningeal surface and to distant parts of the CNS where occur secondary leptomeningeal metastatic deposits. Diffuse covering of the leptomeninges is particularly frequent in hematological malignancies and plaque-like deposits with invasion of the VirchowRobin spaces and nodular formations are more characteristics of solid tumors ${ }^{2,5,6}$.

\section{CSF diagnosis}

Routinely, three methods are used: clinical signs and symptoms, CSF analysis, and magnetic resonance imaging (MRI) of the brain and spine ${ }^{3}$. Clinical manifestations are often subtle and unspecific and MRI sensitivity and specificity differ with the type of primary cancer, location of CNS involvement and may be normal. CSF cytological study has a high specificity (>95\%), but its sensitivity is generally less than $50 \%$, mainly in parenchymal involvement. Concerning to CSF biomarkers, there is still a lack in specificity and sensitivity.

According to the National Comprehensive Cancer Network (NCCN) guidelines, CSF study is fundamental in the diagnosis of CNS and CSF cancer involvement ${ }^{7}$. The guidelines suggest any one of the following diagnostic criteria are sufficient to diagnose LM: CSF positive for tumor cells (positive CSF cytology); radiologic findings in the CNS consistent with LM irrespective of supportive clinical findings or alternatively and more controversial, clinical signs and symptoms consistent with LM and a nonspecific but abnormal CSF analysis (high white blood cell count, low glucose, and elevated protein) in a patient known to have a cancer. In spite of this, underdiagnosis remains a major problem in establishing a diagnosis of LM as specific assessments are required (CSF analysis and CNS imaging) and because CSF cytology and neuraxis imaging are often normal ${ }^{7-9}$.

Primary cancers associated with BM patients are mainly: lung, breast, renal, colorectal cancers and melanoma. Prevalence of BM is estimated at $9 \%$ but it is probably underestimated. Time from initial to BM diagnoses is increasing and BM is occurring more frequently in individuals with advanced-stage disease. Biology of BM remains poorly known. Interactions between circulated tumoral cells (CTC) and blood-brain-barrier (BBB) cells are required. Some cytokines may act as CTC attractants and promote BM formation ${ }^{4}$.

Abnormalities of the standard CSF analysis are observed in more than $90 \%$ of the cases of LM. These abnormalities include increased opening pressure $(>200 \mathrm{~mm}$ of $\mathrm{H} 2 \mathrm{O})$ in $46 \%$, increased leukocytes $\left(>4 / \mathrm{mm}^{3}\right)$ in $57 \%$, elevated protein $(>50 \mathrm{mg} / \mathrm{dl})$ in $76 \%$, and decreased glucose $(<60 \mathrm{mg} / \mathrm{dl})$ in $54 \%$. Although indicative of LM, these CSF abnormalities are nonspecific ${ }^{3,5,6}$.

Morphological detection of tumor cells in CSF cytology or research of soluble factors in CSF or also in blood (tumor markers) is used for diagnosis, tumor staging, and therapy decisions. CSF cytology may be particularly useful for the detection of leptomeningeal spread of some type of cancer as medulloblastoma, lymphoma, and metastatic carcinoma and melanoma. In intracranial germ cell tumours, serum and CSF levels of $\alpha$-fetoprotein and $\beta$-human chorionic gonadotropin are important biomarkers. In patients with primary central nervous system lymphoma, CSF protein and glucose levels, flow cytometry, and immunoglobulin heavy-chain gene rearrangement studies are of diagnostic and prognostic relevance ${ }^{8,9}$. Cerebrospinal fluid cytology can provide definitive diagnostic information in CNS lymphoma and, with the aid of immunohistochemical studies, it has been possible to identify atypical lymphoid cells as monoclonal or neoplastic ${ }^{1-3}$.

The presence of malignant cells in the CSF is diagnostic of LM, but as its true for most cytological analysis, assignment to a particular tumor is often not possible. In patients with positive CSF cytology, up to $45 \%$ will be cytologically negative on first examination ${ }^{8,9}$. In a second tap, the yield is increased to $80 \%$, but little benefit is obtained from repeated lumbar punctures after two punctures ${ }^{8-10}$. Some procedures can improve the sensitivity of the CSF cytological analysis including submission of a nonhemorrhagic CSF specimen. Obtaining large CSF sampling volumes (>10.5 ml) improves the yield of CSF sensitivity ${ }^{11}$. The sensitivity of CSF cytology increased from $68 \%$ to $97 \%$ for 3.5 and $10.5 \mathrm{ml}$ samples, respectively. Processing of CSF specimens in a timely manner is also critical to improve the sensitivity of CSF cytology. The viability of cells depends on time between sampling and laboratory examination ${ }^{9,11}$. After 30 minutes, $50 \%$ of the cells remain viable, and only $10 \%$ of cells remain viable after 90 minutes $^{9,11}$. The low sensitivity of CSF cytology makes it difficult not only to diagnose LM, but also to assess response to treatment ${ }^{11}$.

Biochemical markers, immunohistochemistry and molecular biology techniques applied to CSF have been explored in an attempt to find a reliable biological marker of disease $^{3,6,11}$. Numerous biochemical markers have been evaluated, for early diagnosis and to effectiveness of treatment evaluation ${ }^{12-14}$. In general, their use has been limited by poor sensitivity and specificity. These biomarkers may be nonspecific, such as $\beta$-glucuronidase, lactate dehydrogenase, beta2microglobulin, carcinoembryonic antigen or alternatively. CSF release of tumor biomarkers markers has been demonstrated in many patients with LM, however, there was no clear correlation with the type of carcinoma or response to treatment observed ${ }^{12,13,14}$. Particular organ-specific tumor markers such as CA 15-3, CA 125, CA 19-9, CA724, AFP, NSE, Cyfra 21-1, EGFR, a-fetoprotein, b-human chorionic gonadotropin can be relatively specific for LM when elevated in CSF in the absence of markedly elevated serum levels ${ }^{6,11-14}$. Nonspecific tumor markers such as molecules involved in CNS penetration (eg, matrix metalloproteinases and cathepsins), tumor cell tropism (eg, chemokines CXCL8 and CCL18) and vascular endothelial growth factor can be strong indirect indicators 
of LM but none are sensitive enough to improve the cytological diagnosis ${ }^{3,6,11,17}$. CSF antithrombin III levels have been suggested as a useful biomarker in patients with primary CNS lymphoma, but have not been evaluated in patients with lymphomatous meningitis ${ }^{6,15}$. Emerging biomarkers for LM such as proangiogenic molecules (vascular endothelial growth factor [VEGF], urokinase plasminogen activator [uPA], and tissue plasminogen activator [tPA]) have also been evaluated. In the majority of studies, VEGF levels were increased in patients with LM, but sensitivities (51.4-100\%) and specificities (71-100\%) have widely varied. Combinations of different markers have been suggested to increase the sensitivity of CSF biomarkers in $\mathrm{LM}^{6,10}$. Profiling CSF proteins and in particular those involved in the metastatic process, may have potential diagnostic and prognostic value. CXCL13 and CXCL12 mediate chemotaxis of CNS lymphoma cells, and CXCL13 concentration in CSF is a prognostic marker ${ }^{17}$. The use of these biochemical markers can be helpful as adjunctive diagnostic tests and response to treatment assessment ${ }^{6,11}$. Microglobulin is an HLA antigen-associated cell surface protein for which elevation reflects increased cell turnover. Although serum levels are not able to distinguish systemic versus CNS involvement, CSF $\beta 2$-microglobulin has been correlated with the presence of CNS disease in leukemia and lymphoma in small numbers of patients, as well as response to therapy. Levels of CSF $\beta 2$-microglobulin may be elevated up to $50 \%$ in patients with leukemia or lymphoma and CSF involvement ${ }^{14,16}$. Malignant brain tumors may show an increased fraction of anaerobic LDH concentrations (LD4 and LD5) in $\mathrm{CSF}^{16}$.

When added to the determination of cell count and protein, oligoclonal bands testing contributes to the sensitivity of CSF analysis in diagnosing paraneoplastic syndromes ${ }^{14,15}$.

Cerebrospinal fluid flow cytometry is a useful adjunct to CSF cytology and has been shown to increase the ability to detect CNS involvement in high-risk individuals, particularly in hematologic tumors ${ }^{9,15}$. Cytology examines morphologic patterns, and flow cytometry has the potential to provide information about the immunophenotype of the lymphocytes (by surface protein expression) and up to $80 \%$ of lymphoma cases with CSF involvement are detected on the first CSF sample ${ }^{11,16}$. When CSF flow cytometry is suspicious for lymphoma, a matching abnormal immunophenotype in a blood or bone marrow sample supports a concurrent systemic lymphoma diagnosis. Rarely, a false-positive CSF result may occur if a CSF sample is contaminated by peripheral blood lymphocytes in an individual with active systemic lymphoma ${ }^{9,11,15}$.

Flow cytometry provides information about cell surface protein expression, and immunoglobulin heavy chain (IgH) rearrangement testing analyzes the clonality of the antibodies being produced. Using PCR analysis of CSF, IgH rearrangement studies amplify the CDR-3 region of the IgH. In cases of a neoplastic proliferation of lymphocytes, a unique variable/diversity/joining arrangement is produced, resulting in a single sharp band on agarose gel. In contrast, nonneoplastic proliferation of lymphocytes as seen in inflammatory processes produces a wide band reflecting multiple different heavy chain sequences. IgH rearrangement analysis have a sensitivity of $58 \%$ and a specificity of $85 \%$ in the detection of monoclonal antibody production. Pretreatment with corticosteroids reduces the sensitivity of IgH rearrangement studies as well as the sensitivity of cytological analysis ${ }^{16,17}$.

DNA single cell cytometry, techniques that measure the chromosomal content of cells, and fluorescence in-situ hybridization (FISH), that detects numerical and structural genetic aberrations as a sign of malignancy, can give additional diagnostic information, but still have a low sensitivity. Polymerase chain reaction (PCR) can establish a correct diagnosis when cytology is inconclusive, but the genetic alteration of the neoplasia must be known for it to be amplified with this technique, and this is generally not the case, particularly in solid tumors ${ }^{15,16}$.

The use of monoclonal antibodies for immunohistochemical analysis in leptomeningeal metastasis does not significantly increase the sensitivity of cytology alone; however, in the case of leukemia and lymphoma, antibodies against surface markers can be used to distinguish between reactive and neoplastic lymphocytes in the $\mathrm{CSF}^{4,8}$.

Proteomic analysis of CSF has revealed numerous proteins that are differentially expressed in CNS lympho$\mathrm{ma}^{6,11,15,16}$. Among these, antithrombin III, a serine protease inhibitor that is associated with neovascularization in CNS lymphoma, has been prospectively validated. Elevated levels of antithrombin III were found in the CSF of patients with CNS lymphoma compared with the CSF of patients with other cancers and those with inflammatory neurologic conditions. Elevated antithrombin III levels correlated with a shorter overall survival and less response to chemotherapy. Antithrombin III levels higher than $1.2 \mathrm{~g} / \mathrm{mL}$ made possible the detection of CNS lymphoma with $75 \%$ sensitivity and 98.7\% specificity ${ }^{6,11,15,16}$

MicroRNAs are short, nontranslated fragments of RNA that bind to 3' untranslated regions of messenger RNA and repress protein translation in several molecular pathways. MicroRNAs may function as oncogenes or tumor suppressor genes, and abnormal expression of microRNAs has been associated with several different cancers, including lymphoma $a^{3,9,10,16}$.

The molecular constituents of CSF can be applied as biomarkers to facilitate diagnosis of pathological processes within brain. At least 2 types of CSF biomarkers are used routinely: Epstein-Barr viral DNA in the diagnosis of AIDSrelated CNS lymphoma and the germ cell markers a-fetoprotein and/or b-human chorionic gonadotropin in the 
diagnosis of childhood CNS germinoma. In the proper context, detection of high concentrations of these biomarkers in CSF may eliminate the need for brain biopsy ${ }^{9,10}$.

Epstein-Barr virus (EBV) activity has been associated with the development of CNS lymphoma in both HIV and posttransplant lymphoproliferative disorder. Epstein-Barr virus polymerase chain reaction (PCR) on CSF has been proposed as a useful diagnostic tool to identify cases of active
EBV infection and to distinguish lymphoma from infectious encephalitides. The sensitivity of EBV PCR ranges from $80 \%$ to $100 \%$, with a specificity of $93 \%$ to $100 \%^{9,16}$. In posttransplant lymphoproliferative disorder, $93 \%$ of biopsy-confirmed cases also had positive CSF EBV PCR results. In routine clinical use, however, the positive predictive value of CSF EBV PCR may be considerably lower when attempting to discriminate lymphoma from CNS toxoplasmosis ${ }^{9,16}$.

\section{References}

1. Ramirez C, Blonski M, Belin C, Carpentier A, Taillia H. Brain metastasis: clinical and cognitive assessments. Bull Cancer 2013;100:83-88.

2. Lakshmi Nayak L, Eudocia Quant Lee EQ, Wen PY. Epidemiology of brain metastases. Curr Oncol Rep 2012;14:48-54.

3. Chamberlain MC, Glantz M, Groves MD, Wilson WH. Diagnostic tools for neoplastic meningitis: detecting disease, identifying patient risk, and determining benefit of treatment. Sem Oncol 2009;36(Suppl): S35-S45.

4. Tabouret E, Bauchet L, Carpentier AF. Brain metastases epidemiology and biology. Bull Cancer 2013;100:57-62.

5. Gleissner B, Chamberlain MC. Neoplastic meningitis. Lancet Neurol 2006;5: 443-452.

6. Rhun EL, Taillibert S, Marc C, Chamberlain MC. Carcinomatous meningitis: leptomeningeal metastases in solid tumors. Surg Neurol Int 2013;4(Suppl 4): S265-S288.

7. Brem SS, Bierman PJ, Brem $\mathrm{H}$, et al. National Comprehensive Cancer Network, Central nervous system. J Natl Compr Canc Netw 2011:9:352-400.

8. Chamberlain MC. Leptomeningeal metastasis. Curr Opin Oncol 2010; 22:627-635.

9. Scott BJ, Douglas VC, Tihan T, Rubenstein JL, Josephson SA. A Systematic approach to the diagnosis of suspected central nervous system lymphoma. JAMA Neurol 2013;70:311-319.

10. Groves MD, Hess KR, Puduvalli VK, et al. Biomarkers of disease: cerebrospinal fluid vascular endothelial growth factor (VEGF) and stromal cell derived factor (SDF)-1 levels in patients with neoplastic meningitis (NM) due to breast cancer, lung cancer and melanoma. J Neurooncol 2009;94:229-234.

11. Chamberlain MC, Johnston SK. Neoplastic meningitis: survival as a function of cerebrospinal fluid cytology. Cancer 2009;5:1941-1946.

12. Corsini E, Bernardi G, Gaviani P, et al. Intrathecal synthesis of tumor markers is a highly sensitive test in the diagnosis of leptomeningeal metastasis from solid cancers. Clin Chem Lab Med 2009;47:874-879.

13. Kang SJ, Kim KS, Ha YS, et al. Diagnostic value of cerebrospinal fluid level of carcinoembryonic antigen in patients with leptomeningeal carcinomatous metastasis. J Clin Neurol 2010;6:33-37.

14. Bougel S, Lhermitte B, Gallagher G, et al. Methylation of the hTERT promoter: a novel cancer biomarker for leptomeningeal metastasis detection in cerebrospinal fluids. Clin Cancer Res 2013;19:2216-2223.

15. Huttner A. Overview of primary brain tumors pathologic classification, epidemiology, molecular biology, and prognostic markers. Hematol Oncol Clin N Am 2012;26:715-732.

16. Galati D, Di Noto R, Del Vecchio L. Diagnostic strategies to investigate cerebrospinal fluid involvement in haematological malignancies. Leuk Res 2013;37:231-237.

17. Rubenstein JL, Wong VS, Kadoch C, et al. CXCL13 plus IL-10 is highly specific for the diagnosis of CNS lymphoma. Blood 2013;121: 4740-4748. 